\title{
The combination of ground-based astrometric compilation catalogues with the HIPPARCOS Catalogue
}

\section{Long-term predictions and short-term predictions}

\begin{abstract}
R. Wielen, H. Lenhardt, H. Schwan, and C. Dettbarn
Astronomisches Rechen-Institut, Moenchhofstrasse 12-14, 69120 Heidelberg, Germany

Received 4 August 2000 / Accepted 4 December 2000

Abstract. The combination of ground-based astrometric compilation catalogues, such as the FK5 or the GC, with the results of the ESA Astrometric Satellite HIPPARCOS produces for many thousands of stars proper motions which are significantly more accurate than the proper motions derived from the HIPPARCOS observations alone. In Paper I (Wielen, et al. 1999, A\&A, 347, 1046) we have presented a method of combination for single stars (SI mode). The present Paper II derives a combination method which is appropriate for an ensemble of "apparently single-stars" which contains undetected astrometric binaries. In this case the quasi-instantaneously measured HIPPARCOS proper motions and positions are affected by "cosmic errors", caused by the orbital motions of the photo-centers of the undetected binaries with respect to their center-of-mass. In contrast, the ground-based data are "mean values" obtained from a long period of observation. We derive a linear "long-term prediction" (LTP mode) for epochs far from the HIPPARCOS epoch $T_{\mathrm{H}} \sim 1991.25$, and a linear "short-term prediction" (STP mode) for epochs close to $T_{\mathrm{H}}$. The most accurate prediction for a position at an arbitrary epoch is provided by a smooth, non-linear transition from the STP solution to the LTP solution. We present an example for the application of our method, and we discuss the error budget of our method for the FK6 (a combination of the FK5 with HIPPARCOS) and for the combination catalogue GC+HIP. For the basic fundamental stars, the accuracy of the FK6 proper motions in the LTP mode is better than that of the HIPPARCOS proper motions (taking here the cosmic errors into account) by a factor of more than 4 .
\end{abstract}

Key words. astrometry - catalogs - stars: binaries: general

\section{Introduction}

In Paper I (Wielen et al. 1999b), we have shown that the combination of the data of the HIPPARCOS astrometric satellite (ESA 1997) with ground-based results (such as the FK5) is able to provide for many stars individual proper motions which are significantly more accurate than the HIPPARCOS proper motions themselves. The method has been already successfully applied in the construction of the FK6, the Sixth Catalogue of Fundamental Stars (Part I of the FK6: Wielen et al. 1999d, Part III of the FK6: Wielen et al. 2000a).

The method of combination presented in Paper I is, however, strictly valid for single stars only. In the FK6, we call this procedure therefore the "single-star mode".

In reality most of the stars are members of binaries or of multiple systems. If the duplicity of an individual object is already definitely known, either from ground-based investigations or from HIPPARCOS observations, then the method of combination has to be changed properly in

Send offprint requests to: R. Wielen,

e-mail: wielen@ari.uni-heidelberg.de order to obtain meaningful results. We call such procedures "special solutions". Paper III of this series of papers will discuss the special solutions for visual binaries and other types of double stars.

The present paper (Paper II) describes an appropriate method of the combination of HIPPARCOS results with ground-based observations for "apparently single stars". Even if we have removed from such a sample of apparently single stars all the objects with known duplicity, there remains beside the truly single-stars a large number of hitherto undetected astrometric binaries. The measured photo-center of an unresolved astrometric binary moves on the sky on a wavy curve, in contrast to the linear motion of single stars (Fig. 1). In such a case, an "instantaneously" measured proper motion deviates from a "mean" proper motion, averaged over a long interval of time. The proper motions provided by HIPPARCOS (ESA 1997) are essentially such instantaneously measured quantities, since they are derived from positional measurements spread over about three years only. In contrast, the proper motions given in the FK5 (Fricke et al. 1988, 1991) are long-term averages over up to about 200 years. 




Time $\mathrm{t}$

Fig. 1. Wavy motion of the photo-center of an astrometric binary (solid curve) around the linear motion of its center-ofmass (cms; dashed line). An instantaneous position and an instantaneous proper motion are indicated (filled dot; arrow). The linear prediction based on the instantaneous values is shown as a dotted line. The long-term averaged "mean" proper motion is equal to the motion of the $\mathrm{cms}$

We have called the difference between the instantaneous proper-motion and the mean one the "cosmic error" of the instantaneous proper motion (Wielen 1995a,b, 1997; Wielen et al. 1997).

In some cases, the individual cosmic error is so large that the duplicity of an apparently single star is strongly indicated by this fact. We have called such objects " $\Delta \mu$ binaries" (Wielen et al. 1999a). In most cases, however, the cosmic error in the HIPPARCOS proper motion of a hitherto undetected astrometric binary is not individually significant, but can be shown to exist only statistically in a larger sample of apparently single stars. Nevertheless, an appropriate combination method should not neglect the statistical consequences of these cosmic errors. Our comparison of HIPPARCOS proper motions with groundbased results has shown that, at least for brighter stars, the average cosmic error in a HIPPARCOS proper motion is often larger than the HIPPARCOS measuring error, typically by a factor of three (Wielen 1995a,b; Wielen et al. 1997, 1998, 1999c).

Wielen (1997, henceforth called Paper WPSA) has developed a coherent scheme of "statistical astrometry" for handling the effects of cosmic errors in high-precision astrometry. In the following sections, we shall apply the concepts of statistical astrometry to the problem of combining the HIPPARCOS results with ground-based measurements for a sample of apparently single stars. The main results will be solutions which we call the "long-term prediction mode (LTP)" and the "short-term prediction mode (STP)". Our results have already been applied for the LTP and STP solutions given in Part I and Part III of the FK6 (Wielen et al. 1999d, 2000a).

It is clear that in principle the most desirable solution for our problem would be to treat each star individually and fully correctly. This would mean (1) for truly single stars: to use the "single-star mode (SI)" described in Paper I, and (2) for binaries: to apply individual orbital corrections, as e.g. done for Polaris by Wielen et al. (2000b). However, since for "apparently single stars" the true nature (single or double) of the individual objects is unknown, we have to rely on statistical methods in order to handle such a sample of stars properly. The treatment of a sample of apparently single stars by our statistical procedures gives on average the best astrometric prediction, and it provides the most realistic error budget for such a sample. In this sense, our statistical treatment is certainly much more appropriate than to ignore the binary nature of a large fraction of a sample of apparently single stars altogether.

In Paper I, we have already pointed out that the older observations carry a high weight in the combination of ground-based measurements with HIPPARCOS data, and that therefore the GC (Boss et al. 1937) should be also considered here because of its high number of rather wellmeasured stars. We call the result of the combination of the GC with HIPPARCOS the combination catalogue $\mathrm{GC}+\mathrm{HIP}$.

\section{Basic concepts and equations}

\subsection{Concepts}

With respect to the two catalogues which should be combined into a new one, we follow closely the situation described in Sect. 2 of Paper I. We are using also as far as possible the nomenclature of that section.

We assume that two astrometric compilation catalogues are available, identified by the indices 1 (e.g. for the FK5) and 2 (e.g. for HIPPARCOS). For the combined catalogue (e.g. the FK6), we use the index C, usually now supplemented by an additional subindex which identifies the special mode of the solution (e.g. LTP or STP). Each of the two basic catalogues $(i=1,2)$ provides for the stars a position $x_{i}\left(T_{i}\right)$ at a central epoch $T_{i}$ and a proper motion $\mu_{i}$ for two angular coordinates (e.g. right ascension $\alpha_{*}=\alpha \cos \delta$ and declination $\delta$ ). The mean measuring errors of $x_{i}\left(T_{i}\right)$ and $\mu_{i}$ are denoted by $\varepsilon_{x, i}$ and $\varepsilon_{\mu, i}$. Usually one of the catalogues (e.g. $i=1$ ) has first to be reduced to the astrometrical system of the other catalogue (e.g. the FK5 to the HIPPARCOS system). In this case, $x_{1}$ and $\mu_{1}$ denote the already systematically corrected quantities, and their mean errors $\varepsilon_{x, 1}$ and $\varepsilon_{\mu, 1}$ include the uncertainty of the systematic corrections. For the determination of the systematic differences between two catalogues, we use methods developed for the construction of the FK5 (Bien et al. 1978). 
With respect to the principles of statistical astrometry, we make now the important additional assumptions that Catalogue 1 gives "mean" quantities for $x$ and $\mu$, averaged over a long interval of time, while Catalogue 2 provides "instantaneously" measured values of $x$ and $\mu$. If we apply our scheme to a combination of the FK5 with HIPPARCOS, both assumptions are fulfilled to a high degree of approximation: the FK5 is based on groundbased observations spread over about two centuries, while the HIPPARCOS results are obtained from measurements made during a short interval of time, about three years only. In the terminology of statistical astrometry, our assumptions mean that we suppose that the FK5 is free from cosmic errors. The cosmic errors in the HIPPARCOS positions and proper motions are denoted by $c_{x}=(\xi(0))^{1 / 2}$ and $c_{\mu}=(\eta(0))^{1 / 2}$. The correlation functions $\xi(\Delta t)$, $\eta(\Delta t)$, and $\zeta(\Delta t)$ are explained in WPSA (especially Sect. 3). Numerical values for $c_{x}(p)$ and $c_{\mu}(p)$ as functions of the parallax $p$ will be provided in Sect. 5 .

\subsection{Basic equations}

In the combination of two astrometric catalogues of which at least one contains instantaneously measured data (affected by cosmic errors), the predicted position $x_{\mathrm{p}}(t)$ at an arbitrary epoch $t$ should not be anymore a linear function of time. According to the principles of statistical astrometry, the "best" prediction $x_{\mathrm{p}}(t)$ for the true instantaneous position $x(t)$ is given by the non-linear expression (WPSA, Eq. (82), with a slight change in nomenclature):

$$
\begin{aligned}
x_{\mathrm{p}}(t)= & x_{1}\left(T_{1}\right)+\mu_{1}\left(t-T_{1}\right) \\
+ & \gamma(t)\left(x_{2}\left(T_{2}\right)-x_{1}\left(T_{1}\right)-\mu_{1}\left(T_{2}-T_{1}\right)\right) \\
+ & \beta(t)\left(\mu_{2}\left(T_{2}\right)-\mu_{1}\right)\left(t-T_{2}\right) .
\end{aligned}
$$

The quantities $\gamma(t)$ and $\beta(t)$ are functions of time $t$ to be determined. For computational reasons, we often use

$B(t)=\beta(t)\left(t-T_{2}\right)$

instead of $\beta(t)$.

The functions $\gamma(t)$ and $\beta(t)$ are determined from the condition that the mean error $\varepsilon_{x, \mathrm{p}}(t)$ of the predicted positions $x_{\mathrm{p}}(t)$ should be a minimum for every value of $t$, averaged over the ensemble:

$\varepsilon_{x, \mathrm{p}}^{2}(t)=<\left(x_{\mathrm{p}}(t)-x(t)\right)^{2}>=\min$.

The operator $\langle q\rangle$ means, as in WPSA (1997), the average of the quantity $q$ over the ensemble of similar stars. For an individual star, $\langle q\rangle$ can be interpreted as the "expectation value" of the quantity $q$ for this star. In this sense, we consider the ensemble averages as statistical predictions for the mean behaviour of a typical individual member of the ensemble.

Inserting Eq. (1) into Eq. (3), and using the scheme of statistical astrometry, we obtain for $\varepsilon_{x, \mathrm{p}}^{2}(t)$ :

$\varepsilon_{x, \mathrm{p}}^{2}(t)=<\left(\left(x_{\mathrm{p}}(t)-x_{\mathrm{m}}(t)\right)-\left(x(t)-x_{\mathrm{m}}(t)\right)\right)^{2}>$

$$
\begin{aligned}
=< & \left(\left(x_{1}\left(T_{1}\right)-x_{\mathrm{m}}\left(T_{1}\right)\right)(1-\gamma(t))\right. \\
& +\left(\mu_{1}-\mu_{\mathrm{m}}\right)\left(t-T_{1}-\gamma(t)\left(T_{2}-T_{1}\right)-\beta(t)\left(t-T_{2}\right)\right) \\
& +\gamma(t)\left(\left(x_{2}\left(T_{2}\right)-x\left(T_{2}\right)\right)+\left(x\left(T_{2}\right)-x_{\mathrm{m}}\left(T_{2}\right)\right)\right) \\
& +\beta(t)\left(t-T_{2}\right)\left(\left(\mu_{2}\left(T_{2}\right)-\mu\left(T_{2}\right)\right)+\left(\mu\left(T_{2}\right)-\mu_{\mathrm{m}}\right)\right) \\
& \left.-\left(x(t)-x_{\mathrm{m}}(t)\right)\right)^{2}> \\
=( & \left.+(\gamma(t))^{2}\right) \xi(0)-2 \gamma(t) \xi\left(t-T_{2}\right) \\
& +\left(\beta(t)\left(t-T_{2}\right)\right)^{2} \eta(0)-2 \beta(t)\left(t-T_{2}\right) \zeta\left(t-T_{2}\right) \\
& +\varepsilon_{x, 1}^{2}(1-\gamma(t))^{2}+\varepsilon_{x, 2}^{2}(\gamma(t))^{2} \\
& +\varepsilon_{\mu, 1}^{2}\left(t-T_{1}-\gamma(t)\left(T_{2}-T_{1}\right)-\beta(t)\left(t-T_{2}\right)\right)^{2} \\
& +\varepsilon_{\mu, 2}^{2}\left(\beta(t)\left(t-T_{2}\right)\right)^{2} .
\end{aligned}
$$

The quantities $x_{\mathrm{m}}(t)$ and $\mu_{\mathrm{m}}=\dot{x}_{\mathrm{m}}$ are the true mean position and the true mean proper motion of the star (in the sense of statistical astrometry). $x_{\mathrm{m}}$ is a linear function of $t$, e.g.

$x_{\mathrm{m}}(t)=x_{\mathrm{m}}\left(T_{1}\right)+\mu_{\mathrm{m}}\left(t-T_{1}\right)$.

In deriving Eq. (4) we have used the following relations: According to our assumption that Catalogue 1 provides mean quantities, $x_{1}\left(T_{1}\right)$ and $\mu_{1}$ differ from $x_{\mathrm{m}}\left(T_{1}\right)$ and $\mu_{\mathrm{m}}$ by their measuring errors only:

$$
\begin{aligned}
<\left(x_{1}\left(T_{1}\right)-x_{\mathrm{m}}\left(T_{1}\right)\right)^{2}> & =\varepsilon_{x, 1}^{2}, \\
<\left(\mu_{1}-\mu_{\mathrm{m}}\right)^{2}> & =\varepsilon_{\mu, 1}^{2} .
\end{aligned}
$$

The quantities $x(t)$ and $\mu(t)$ are the true instantaneous position and proper motion of the star at epoch $t$. Since Catalogue 2 is assumed to provide instantaneous quantities, $x_{2}\left(T_{2}\right)$ and $\mu_{2}\left(T_{2}\right)$ differ from $x\left(T_{2}\right)$ and $\mu\left(T_{2}\right)$ also by their measuring errors only:

$<\left(x_{2}\left(T_{2}\right)-x\left(T_{2}\right)\right)^{2}>=\varepsilon_{x, 2}^{2}$,

$<\left(\mu_{2}\left(T_{2}\right)-\mu\left(T_{2}\right)\right)^{2}>=\varepsilon_{\mu, 2}^{2}$.

The correlation functions $\xi(\Delta t), \eta(\Delta t)$, and $\zeta(\Delta t)$ (see WPSA) occur because of:

$$
\begin{aligned}
& <\left(x\left(T_{2}\right)-x_{\mathrm{m}}\left(T_{2}\right)\right)^{2}>=<\left(x(t)-x_{\mathrm{m}}(t)\right)^{2}>=\xi(0) \\
& <\left(x\left(T_{2}\right)-x_{\mathrm{m}}\left(T_{2}\right)\right)\left(x(t)-x_{\mathrm{m}}(t)\right)>=\xi\left(t-T_{2}\right) \\
& <\left(\mu\left(T_{2}\right)-\mu_{\mathrm{m}}\right)^{2}>=\eta(0) \\
& <\left(x(t)-x_{\mathrm{m}}(t)\right)\left(\mu\left(T_{2}\right)-\mu_{\mathrm{m}}\right)>=\zeta\left(t-T_{2}\right)
\end{aligned}
$$

We have further to remember

$<\left(x\left(T_{2}\right)-x_{\mathrm{m}}\left(T_{2}\right)\right)\left(\mu\left(T_{2}\right)-\mu_{\mathrm{m}}\right)>=\zeta(0)=0$.

The products of the other terms occuring in the second line of Eq. (4) all vanish, because the measuring errors are not correlated with the cosmic errors, for example

$<\left(x_{1}\left(T_{1}\right)-x_{\mathrm{m}}\left(T_{1}\right)\right)\left(x(t)-x_{\mathrm{m}}(t)\right)>=0$, 
and because the measuring errors of $x_{i}\left(T_{i}\right)$ and $\mu_{i}\left(T_{i}\right)$ are not correlated at the central epoch $T_{i}$ (by definition of the central epoch), for example:

$<\left(x_{1}\left(T_{1}\right)-x_{\mathrm{m}}\left(T_{1}\right)\right)\left(\mu_{1}-\mu_{\mathrm{m}}\right)>=0$,

and finally because the measuring errors of $x_{1}$ and $\mu_{1}$ are not correlated with those of $x_{2}$ and $\mu_{2}$, for example

$<\left(x_{1}\left(T_{1}\right)-x_{\mathrm{m}}\left(T_{1}\right)\right)\left(\mu_{2}\left(T_{2}\right)-\mu\left(T_{2}\right)\right)>=0$.

The conditions for the minimum of $\varepsilon_{x, \mathrm{p}}^{2}(t)$ with respect to $\gamma$ and $\beta$ are

$\frac{\partial \varepsilon_{x, \mathrm{p}}^{2}}{\partial \gamma}=0$

and

$\frac{\partial \varepsilon_{x, \mathrm{p}}^{2}}{\partial \beta}=0$

Carrying out these procedures and using the final part of Eq. (4) for $\varepsilon_{x, \mathrm{p}}^{2}(t)$, we obtain the following two equations of condition for the functions $\gamma(t)$ and $\beta(t)$ which give the "best" values of $\varepsilon_{x, \mathrm{p}}(t)$ :

$$
\begin{gathered}
\gamma(t)\left(\xi(0)+\varepsilon_{x, 1}^{2}+\varepsilon_{x, 2}^{2}+\varepsilon_{\mu, 1}^{2}\left(T_{2}-T_{1}\right)^{2}\right) \\
+\beta(t)\left(t-T_{2}\right) \varepsilon_{\mu, 1}^{2}\left(T_{2}-T_{1}\right) \\
=\xi\left(t-T_{2}\right)+\varepsilon_{x, 1}^{2}+\varepsilon_{\mu, 1}^{2}\left(t-T_{1}\right)\left(T_{2}-T_{1}\right), \\
\gamma(t) \varepsilon_{\mu, 1}^{2}\left(T_{2}-T_{1}\right)+\beta(t)\left(t-T_{2}\right)\left(\eta(0)+\varepsilon_{\mu, 1}^{2}+\varepsilon_{\mu, 2}^{2}\right) \\
=\zeta\left(t-T_{2}\right)+\varepsilon_{\mu, 1}^{2}\left(t-T_{1}\right) .
\end{gathered}
$$

The Eqs. (20) and (21) correspond to the Eqs. (86) and (87) in WPSA. Solving the Eqs. (20) and (21) for the unknowns $\gamma(t)$ and $\beta(t)$ we obtain:

$$
\begin{aligned}
\gamma(t)= & \left(\left(\xi\left(t-T_{2}\right)+\varepsilon_{x, 1}^{2}\right)\left(\eta(0)+\varepsilon_{\mu, 1}^{2}+\varepsilon_{\mu, 2}^{2}\right)\right. \\
& +\varepsilon_{\mu, 1}^{2}\left(T_{2}-T_{1}\right)\left(\left(\eta(0)+\varepsilon_{\mu, 2}^{2}\right)\left(t-T_{1}\right)\right. \\
& \left.\left.-\zeta\left(t-T_{2}\right)\right)\right) / N
\end{aligned}
$$

$$
\begin{aligned}
B(t)= & \beta(t)\left(t-T_{2}\right) \\
= & \left(\zeta\left(t-T_{2}\right)\left(\xi(0)+\varepsilon_{x, 1}^{2}+\varepsilon_{x, 2}^{2}+\varepsilon_{\mu, 1}^{2}\left(T_{2}-T_{1}\right)^{2}\right)\right. \\
& +\varepsilon_{\mu, 1}^{2}\left(t-T_{1}\right)\left(\xi(0)+\varepsilon_{x, 1}^{2}+\varepsilon_{x, 2}^{2}\right) \\
& \left.-\varepsilon_{\mu, 1}^{2}\left(T_{2}-T_{1}\right)\left(\xi\left(t-T_{2}\right)+\varepsilon_{x, 1}^{2}\right)\right) / N
\end{aligned}
$$

with the auxiliary quantity $N$ :

$$
\begin{aligned}
N= & \left(\xi(0)+\varepsilon_{x, 1}^{2}+\varepsilon_{x, 2}^{2}\right)\left(\eta(0)+\varepsilon_{\mu, 1}^{2}+\varepsilon_{\mu, 2}^{2}\right) \\
& +\varepsilon_{\mu, 1}^{2}\left(T_{2}-T_{1}\right)^{2}\left(\eta(0)+\varepsilon_{\mu, 2}^{2}\right) .
\end{aligned}
$$

For deriving the functions $\gamma(t)$ and $\beta(t)$, we have up to now implicitely assumed that all the stars in the ensemble have the same measuring errors. This is, of course, not strictly true in reality. However, the ensemble averages are actually neccessary for handling the cosmic errors only, but not for treating the measuring errors. Hence we shall

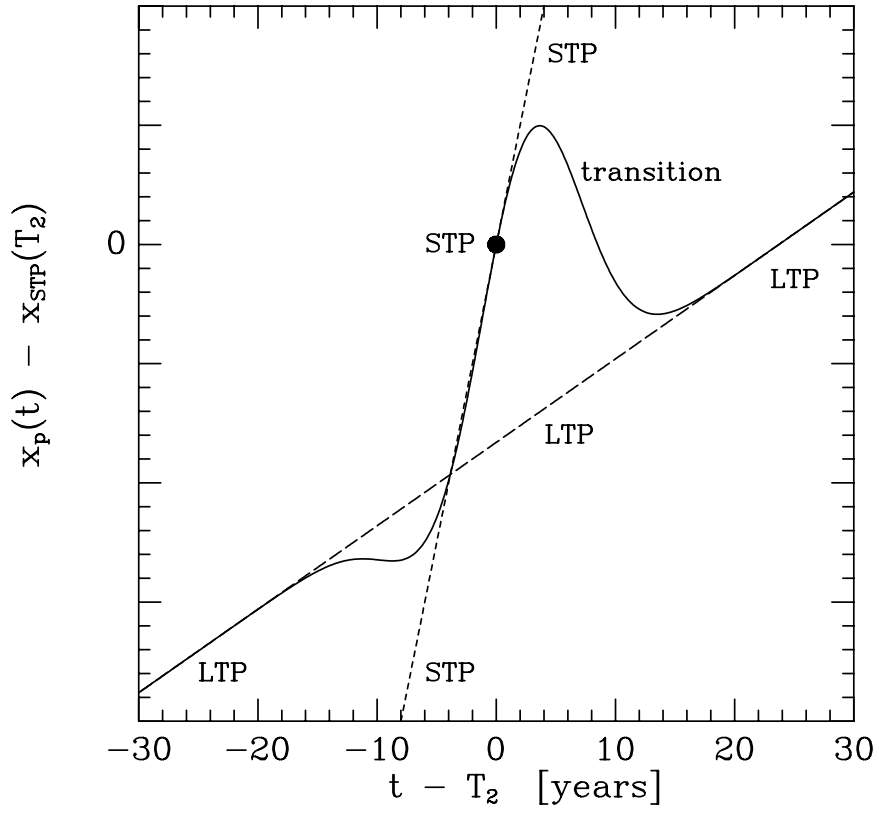

Fig. 2. Schematic illustration of the non-linear transition of the "best" prediction $x_{\mathrm{p}}(t)$ (solid curve) from the linear shortterm prediction $x_{\mathrm{STP}}(t)$ (STP mode, valid for epochs $t \sim T_{2}$; short-dashed line) to the linear long-term prediction $x_{\text {LTP }}(t)$ (LTP mode, valid for epochs $\left|t-T_{2}\right| \rightarrow \infty$; long-dashed line)

use all the equations derived above by inserting the individual measuring errors if we treat individual stars of the ensemble. Formally we may imagine to handle subsamples of stars in which the stars have the overall behaviour with respect to the cosmic errors, but in which the common measuring errors are equal to those of the individual star under consideration. It is a more severe problem that we do not make use of our knowledge of how large the individual cosmic errors in $x$ and $\mu$ are for a given individual object (e.g. $\mu_{2}-\mu_{1}$ ). As discussed in WPSA, this would require "conditioned" correlation functions. Since this information is presently not available, we are treating here the consequences of the cosmic errors on the level of ensemble averages only.

Inserting these results for $\gamma(t)$ and $\beta(t)$ from Eqs. (22) and (23) into Eqs. (1) and (4), we derive the prediction $x_{\mathrm{p}}(t)$ for the instantaneous position $x(t)$ of the star and the mean error $\varepsilon_{x, \mathrm{p}}(t)$ of this prediction. The prediction $x_{\mathrm{p}}(t)$ is a non-linear function of $t$, because the correlation functions $\xi\left(t-T_{2}\right)$ and $\zeta\left(t-T_{2}\right)$, which occur in the formulae for $\gamma(t)$ and $\beta(t)$, are non-linear functions. A typical run of $x_{\mathrm{p}}(t)$ is shown in Fig. 2 .

In order to illustrate the properties of our prediction $x_{\mathrm{p}}(t)$, we consider in the following Sects. 2.3 and 2.4 two limiting cases in which we either neglect the measuring errors or the cosmic errors.

\subsection{Measuring errors neglected}

If we neglect all the measuring errors and set $\varepsilon_{x, 1}\left(T_{1}\right)=$ $\varepsilon_{x, 2}\left(T_{2}\right)=\varepsilon_{\mu, 1}=\varepsilon_{\mu, 2}=0$, then we obtain for 
$\gamma(t)$ and $\beta(t)$

$\gamma_{\mathrm{nme}}(t)=\xi\left(t-T_{2}\right) / \xi(0)$

$B_{\text {nme }}(t)=\beta_{\text {nme }}(t)\left(t-T_{2}\right)=\zeta\left(t-T_{2}\right) / \eta(0)$.

These results were already derived and discussed in WPSA (Sect. 4.2.4 and Fig. 10). The corresponding prediction $x_{\mathrm{p}, \mathrm{nme}}(t)$, shown in Fig. 2 , passes through the point $x_{2}\left(T_{2}\right)$ with the slope $\mu_{2}\left(T_{2}\right)$. Therefore, the measured instantaneous position and proper motion at epoch $T_{2}$ are exactly reproduced by $x_{\mathrm{p}, \mathrm{nme}}(t)$. For $t \rightarrow \pm \infty$, the prediction $x_{\mathrm{p}, \mathrm{nme}}(t)$ approaches asymptotically the mean position $x_{\mathrm{m}}(t)=x_{1}(t)=x_{1}\left(T_{1}\right)+\mu_{1}\left(t-T_{1}\right)$ of the star. The uncertainty $\varepsilon_{x, \mathrm{p}, \mathrm{nme}}(t)$ of the prediction is given by

$\varepsilon_{x, \mathrm{p}, \mathrm{nme}}^{2}(t)=\frac{(\xi(0))^{2}-\left(\xi\left(t-T_{2}\right)\right)^{2}}{\xi(0)}-\frac{\left(\zeta\left(t-T_{2}\right)\right)^{2}}{\eta(0)}$.

We see that $\varepsilon_{x, \mathrm{p}, \mathrm{nme}}$ is zero at the epoch $T_{2}$ and approaches $c_{x}=(\xi(0))^{1 / 2}$ for $t \rightarrow \pm \infty$, since in this limit $x_{\mathrm{p}}(t)$ is equal to $x_{\mathrm{m}}(t)$, and $x_{\mathrm{m}}(t)$ differs from the instantaneous position $x(t)$ by the cosmic error $c_{x}$ on average.

\subsection{Cosmic errors neglected}

If we neglect the cosmic errors and set $\xi=\eta=\zeta=0$, then we obtain

$$
\begin{aligned}
\gamma_{\text {nce }}(t)= & \left(\varepsilon_{x, 1}^{2}\left(\varepsilon_{\mu, 1}^{2}+\varepsilon_{\mu, 2}^{2}\right)\right. \\
& \left.+\varepsilon_{\mu, 1}^{2} \varepsilon_{\mu, 2}^{2}\left(T_{2}-T_{1}\right)\left(t-T_{1}\right)\right) / N_{\text {nce }}, \\
B_{\text {nce }}(t)= & \beta_{\text {nce }}(t)\left(t-T_{2}\right)=\left(-\varepsilon_{x, 1}^{2} \varepsilon_{\mu, 1}^{2}\left(T_{2}-T_{1}\right)\right. \\
& \left.+\left(\varepsilon_{x, 1}^{2}+\varepsilon_{x, 2}^{2}\right) \varepsilon_{\mu, 1}^{2}\left(t-T_{1}\right)\right) / N_{\text {nce }},
\end{aligned}
$$

with

$N_{\text {nce }}=\left(\varepsilon_{x, 1}^{2}+\varepsilon_{x, 2}^{2}\right)\left(\varepsilon_{\mu, 1}^{2}+\varepsilon_{\mu, 2}^{2}\right)+\varepsilon_{\mu, 1}^{2} \varepsilon_{\mu, 2}^{2}\left(T_{2}-T_{1}\right)^{2} .(30$

Equation (29) illuminates one of the advantages of introducing $B(t)=\beta(t)\left(t-T_{2}\right)$ as a substitute for $\beta(t)$. While $B_{\text {nce }}\left(T_{2}\right)$, and in general $B\left(T_{2}\right)$ for non-zero measuring errors, remains finite, the quantity $\beta_{\text {nce }}$, and in general $\beta$, tends towards infinity for $t \rightarrow T_{2}$. Only in some degenerated cases, such as $\varepsilon_{\mu, 1}=0$ or $\varepsilon_{x, 2}=0$ or $\varepsilon_{x, 1} \rightarrow \infty$, the quantity $\beta_{\text {nce, }}$ and in general $\beta$, remains finite for $t \rightarrow T_{2}$.

If we insert $\gamma_{\text {nce }}(t)$ and $B_{\text {nce }}(t)$ into Eq. (1), the corresponding prediction $x_{\mathrm{p}, \mathrm{nce}}(t)$ for the position of the star at an epoch $t$ is now a strictly linear function of $t$, since $\gamma_{\text {nce }}(t)$ and $B_{\text {nce }}(t)$ are linear in $t$.

In order to facilitate the understanding of the behaviour of the prediction $x_{\mathrm{p}, \text { nce }}(t)$, we rewrite $x_{\mathrm{p}, \text { nce }}$ by using the auxiliary quantities $T_{\mathrm{C}, \mathrm{nce}}, x_{\mathrm{C}, \mathrm{nce}}, \mu_{\mathrm{C}, \mathrm{nce}}$. If we insert Eqs. (28) and (29) into Eq. (1), we obtain after some lengthy algebra:

$$
x_{\mathrm{p}, \mathrm{nce}}(t)=x_{\mathrm{C}, \mathrm{nce}}\left(T_{\mathrm{C}, \mathrm{nce}}\right)+\mu_{\mathrm{C}, \text { nce }}\left(t-T_{\mathrm{C}, \text { nce }}\right),
$$

with the auxiliary quantities

$$
\begin{aligned}
T_{\mathrm{C}, \mathrm{nce}} & =\frac{w_{x, 1} T_{1}+w_{x, 2} T_{2}}{w_{x, 1}+w_{x, 2}}, \\
x_{\mathrm{C}, \text { nce }}\left(T_{\mathrm{C}, \text { nce }}\right) & =\frac{w_{x, 1} x_{1}\left(T_{1}\right)+w_{x, 2} x_{2}\left(T_{2}\right)}{w_{x, 1}+w_{x, 2}}, \\
\mu_{\mathrm{C}, \text { nce }} & =\frac{w_{\mu, 1} \mu_{1}+w_{\mu, 2} \mu_{2}+w_{\mu, 0} \mu_{0}}{w_{\mu, 1}+w_{\mu, 2}+w_{\mu, 0}}, \\
\mu_{0} & =\left(x_{2}\left(T_{2}\right)-x_{1}\left(T_{1}\right)\right) /\left(T_{2}-T_{1}\right) .
\end{aligned}
$$

The weights $w$ are given by

$$
\begin{aligned}
& w_{x, 1}=\frac{1}{\varepsilon_{x, 1}^{2}} \\
& w_{x, 2}=\frac{1}{\varepsilon_{x, 2}^{2}} \\
& w_{\mu, 1}=\frac{1}{\varepsilon_{\mu, 1}^{2}} \\
& w_{\mu, 2}=\frac{1}{\varepsilon_{\mu, 2}^{2}}, \\
& w_{\mu, 0}=\frac{1}{\varepsilon_{\mu, 0}^{2}}=\frac{\left(T_{2}-T_{1}\right)^{2}}{\varepsilon_{x, 1}^{2}+\varepsilon_{x, 2}^{2}}
\end{aligned}
$$

Inserting $\gamma_{\text {nce }}(t)$ and $B_{\text {nce }}(t)$ into Eq. (4), we obtain the mean error $\varepsilon_{x, \mathrm{p}, \mathrm{nce}}(t)$ of $x_{\mathrm{p}, \mathrm{nce}}$. Using the form of $x_{\mathrm{p}, \text { nce }}$ as given in Eq. (31) and the auxiliary quantities with the index $\mathrm{C}$, we find

$$
\varepsilon_{x, \mathrm{p}, \mathrm{nce}}^{2}(t)=\varepsilon_{x, \mathrm{C}, \mathrm{nce}}^{2}\left(T_{\mathrm{C}, \mathrm{nce}}\right)+\varepsilon_{\mu, \mathrm{C}, \mathrm{nce}}^{2}\left(t-T_{\mathrm{C}, \mathrm{nce}}\right)^{2},
$$

with

$\varepsilon_{x, \mathrm{C}, \mathrm{nce}}^{2}\left(T_{\mathrm{C}, \mathrm{nce}}\right)=\frac{1}{w_{x, 1}+w_{x, 2}}$

$\varepsilon_{\mu, \mathrm{C}, \mathrm{nce}}^{2}=\frac{1}{w_{\mu, 1}+w_{\mu, 2}+w_{\mu, 0}}$

A comparison of the Eqs. (31)-(42) with the analytic version of the single-star-mode solution of Paper I (Sect. 2, especially Eqs. (19), (23), (30)-(32)) shows that the prediction $x_{\mathrm{p}, \mathrm{nce}}(t)$ and its mean error $\varepsilon_{x, \mathrm{p}, \mathrm{nce}}(t)$ are strictly identical with the single-star-mode solution $x_{\mathrm{SI}}(t)$ and its mean error $\varepsilon_{x, \mathrm{SI}}(t)$. This result is very pleasing, since it proves the internal consistency of our scheme: in the limit of vanishing cosmic errors, the prediction $x_{\mathrm{p}}(t)$ according to Eq. (1) is asymptotically approaching the singlestar mode solution $x_{\mathrm{SI}}(t)$. This result is not apriori selfevident, since our definitions for the "best" solution for predicting $x(t)$ differ at least formally in Paper I and in this Paper II (i.e., Eqs. (5) and (35) of Paper I versus Eq. (3) of Paper II). 


\subsection{Motivation for introducing the long-term and short-term prediction}

The general solution of our combination problem is given in Sect. 2.2. for all epochs $t$. The solution $x_{\mathrm{p}}(t)$ is a nonlinear function of $t$, and requires the knowledge of the correlation functions $\xi(\Delta t)$ and $\zeta(\Delta t)$ as functions of the epoch difference $\Delta t$. At present, we do not have a wellestablished knowledge about the run of $\xi(\Delta t)$ and $\zeta(\Delta t)$. Only the cosmic errors $c_{\mu}=(\eta(0))^{1 / 2}$ and $c_{x}=(\xi(0))^{1 / 2}$ can be empirically determined from the comparison of the FK5 with HIPPARCOS, assuming that the FK5 is giving "mean" quantities.

Even if we would know the run of $\xi(\Delta t)$ and $\zeta(\Delta t)$ as a function of $\Delta t$, the non-linearity of $x_{\mathrm{p}}(t)$ would demand a table of $x_{\mathrm{p}}(t)$ for a sequence of epochs, e.g. for each year, if the user should not have the burden to do the full calculation himself by running a program.

We propose the following solution: The general solution for $x_{\mathrm{p}}(t)$ allows rather easily to obtain two limiting solutions for $\Delta t=t-T_{2} \rightarrow \pm \infty$ and for $\Delta t \rightarrow 0$. We call the solution for $\Delta t \rightarrow \pm \infty$ the "long-term prediction (LTP)", and the solution for $\Delta t \rightarrow 0$ the "short-term prediction (STP)" around the epoch $T_{2}$.

Both the LTP and STP solutions are linear in $t$. They can be therefore given in the usual astrometric style, i.e. as a position at a central epoch and a proper motion, together with their mean errors. The details on the LTP and STP solutions $x_{\mathrm{LTP}}(t)$ and $x_{\mathrm{STP}}(t)$ are given in the Sects. 3 and 4 .

The general solution $x_{\mathrm{p}}(t)$ is a smooth transition from short-term prediction $x_{\mathrm{STP}}(t)$ (for epochs around $T_{2}$ ) to the long-term prediction $x_{\text {LTP }}(t)$ for epochs $t$ far away from $T_{2}$. In Sect. 5 , we will discuss a convenient (but only approximately valid) method to carry out this transition, if we know the run of $\zeta(\Delta t)$. This gives at least a rough indication on the process of transition as a function of the epoch difference $\Delta t$, even if $\zeta(\Delta t)$ is not well-established. If, in the future, $\zeta(\Delta t)$ should be better determined, then our method would allow rather conveniently the (approximate) determination of $x_{\mathrm{p}}(t)$ also for epochs inbetween the validity ranges of $x_{\mathrm{STP}}(t)$ and $x_{\mathrm{LTP}}(t)$.

\section{Long-term prediction (LTP)}

We consider in this section the limiting case of the general solution $x_{\mathrm{p}}(t)$ for $\left|t-T_{2}\right| \rightarrow \pm \infty$. This "long-term prediction" $x_{\mathrm{LTP}}(t)$ is valid for epochs not too close to the epoch $T_{2}$ of the instantaneous Catalogue 2 (i.e. here the HIPPARCOS Catalogue with $\left.T_{2} \sim 1991.25\right)$.

We assume that the epoch difference $\Delta t=t-T_{2}$ is so large that the correlation functions $\xi(\Delta t)$ and $\zeta(\Delta t)$ both vanish. Setting $\xi\left(t-T_{2}\right)=0$ and $\zeta\left(t-T_{2}\right)=0$, we obtain from the general Eqs. (22) and (23) for the LTP solution

$$
\begin{aligned}
\gamma_{\mathrm{LTP}}(t)= & \left(\varepsilon_{x, 1}^{2}\left(\varepsilon_{\mu, 1}^{2}+\left[\varepsilon_{\mu, 2}^{2}+\eta(0)\right]\right)\right. \\
& \left.+\varepsilon_{\mu, 1}^{2}\left[\varepsilon_{\mu, 2}^{2}+\eta(0)\right]\left(T_{2}-T_{1}\right)\left(t-T_{1}\right)\right) / N
\end{aligned}
$$

$$
\begin{aligned}
B_{\mathrm{LTP}}(t)= & \beta_{\mathrm{LTP}}(t)\left(t-T_{2}\right) \\
= & \left(-\varepsilon_{x, 1}^{2} \varepsilon_{\mu, 1}^{2}\left(T_{2}-T_{1}\right)\right. \\
& \left.+\left(\varepsilon_{x, 1}^{2}+\left[\varepsilon_{x, 2}^{2}+\xi(0)\right]\right) \varepsilon_{\mu, 1}^{2}\left(t-T_{1}\right)\right) / N
\end{aligned}
$$

where $N$ is still given by Eq. (24). If we compare the Eqs. (43), (44), and (24) for $\gamma_{\mathrm{LTP}}, B_{\mathrm{LTP}}$, and $N=N_{\mathrm{LTP}}$ with the corresponding Eqs. (28)-(30) for $\gamma_{\text {nce }}, B_{\text {nce }}$, and $N_{\text {nce }}$, we find that they are identical if we replace in the equations for the nce solution the quantity $\varepsilon_{x, 2}^{2}$ by

$\varepsilon_{x, 2, \mathrm{LTP}}^{2}=\varepsilon_{x, 2}^{2}+\xi(0)=\varepsilon_{x, 2}^{2}+c_{x}^{2}$,

and $\varepsilon_{\mu, 2}^{2}$ by

$\varepsilon_{\mu, 2, \mathrm{LTP}}^{2}=\varepsilon_{\mu, 2}^{2}+\eta(0)=\varepsilon_{\mu, 2}^{2}+c_{\mu}^{2}$.

This is very plausible, since the instantaneously measured quantities $x_{2}\left(T_{2}\right)$ and $\mu_{2}\left(T_{2}\right)$ are affected by the cosmic errors $c_{x}$ and $c_{\mu}$. If we add these cosmic errors to the corresponding measuring errors $\varepsilon_{x, 2}$ and $\varepsilon_{\mu, 2}$, we obtain "apparent" measuring errors $\varepsilon_{x, 2, \mathrm{LTP}}$ and $\varepsilon_{\mu, 2, \mathrm{LTP}}$. Since the cosmic errors are not correlated with the measuring errors, the summation has to be done quadratically.

Using this finding we obtain for the long-term prediction

$$
x_{\mathrm{LTP}}(t)=x_{\mathrm{LTP}}\left(T_{\mathrm{LTP}}\right)+\mu_{\mathrm{LTP}}\left(t-T_{\mathrm{LTP}}\right),
$$

with

$$
\begin{aligned}
T_{\mathrm{LTP}} & =\frac{w_{x, 1} T_{1}+w_{x, 2, \mathrm{LTP}} T_{2}}{w_{x, 1}+w_{x, 2, \mathrm{LTP}}}, \\
x_{\mathrm{LTP}}\left(T_{\mathrm{LTP}}\right) & =\frac{w_{x, 1} x_{1}\left(T_{1}\right)+w_{x, 2, \mathrm{LTP}} x_{2}\left(T_{2}\right)}{w_{x, 1}+w_{x, 2, \mathrm{LTP}}}, \\
\mu_{\mathrm{LTP}} & =\frac{w_{\mu, 1} \mu_{1}+w_{\mu, 2, \mathrm{LTP}} \mu_{2}+w_{\mu, 0, \mathrm{LTP}} \mu_{0}}{w_{\mu, 1}+w_{\mu, 2, \mathrm{LTP}}+w_{\mu, 0, \mathrm{LTP}}} .
\end{aligned}
$$

The weights $w$ are given by Eqs. (36) and (38), and by

$$
\begin{aligned}
& w_{x, 2, \mathrm{LTP}}=\frac{1}{\varepsilon_{x, 2}^{2}+c_{x}^{2}}, \\
& w_{\mu, 2, \mathrm{LTP}}=\frac{1}{\varepsilon_{\mu, 2}^{2}+c_{\mu}^{2}}, \\
& w_{\mu, 0, \mathrm{LTP}}=\frac{\left(T_{2}-T_{1}\right)^{2}}{\varepsilon_{x, 1}^{2}+\varepsilon_{x, 2}^{2}+c_{x}^{2}} .
\end{aligned}
$$

The long-term prediction $x_{\mathrm{LTP}}(t)$ has in fact two conceptionally different properties: (1) As described above, it is the limit of the general prediction $x_{\mathrm{p}}(t)$ for the true instantaneous position $x(t)$ for $t \rightarrow \pm \infty$. (2) On the other hand, $x_{\operatorname{LTP}}(t)$ is for all epochs $t$ the best prediction for the mean position $x_{\mathrm{m}}(t)$ of the object. This means especially that $\mu_{\mathrm{LTP}}$ is the best estimate of the center-of-mass velocity of the object.

The two different concepts produce two different error estimates $\varepsilon_{x, \mathrm{LTP}}(t)$ for $x_{\mathrm{LTP}}(t)$. If we consider $x_{\mathrm{LTP}}$ as the prediction for the mean position $x_{\mathrm{m}}(t)$ then the mean error $\varepsilon_{x, \mathrm{LTP}, \mathrm{m}}(t)$ is given by:

$\varepsilon_{x, \mathrm{LTP}, \mathrm{m}}^{2}(t)=\varepsilon_{x, \mathrm{LTP}, \mathrm{m}}^{2}\left(T_{\mathrm{LTP}}\right)+\varepsilon_{\mu, \mathrm{LTP}, \mathrm{m}}^{2}\left(t-T_{\mathrm{LTP}}\right)^{2}$, 
with

$\varepsilon_{x, \mathrm{LTP}, \mathrm{m}}^{2}\left(T_{\mathrm{LTP}}\right)=\frac{1}{w_{x, 1}^{2}+w_{x, 2, \mathrm{LTP}}^{2}}$,

$\varepsilon_{\mu, \mathrm{LTP}, \mathrm{m}}^{2}=\frac{1}{w_{\mu, 1}^{2}+w_{\mu, 2, \mathrm{LTP}}^{2}+w_{\mu, 0, \mathrm{LTP}}^{2}}$.

If we consider $x_{\text {LTP }}$ as the prediction for the instantaneous position $x(t)$ for large values of $|\Delta t|=\left|t-T_{2}\right|$, then the uncertainty $\varepsilon_{x, \mathrm{LTP}, \text { ins }}(t)$ of $x_{\mathrm{LTP}}(t)$ is given by

$\varepsilon_{x, \mathrm{LTP}, \mathrm{ins}}^{2}(t)=\varepsilon_{x, \mathrm{LTP}, \mathrm{m}}^{2}(t)+c_{x}^{2}$.

Similarly, the mean error $\varepsilon_{\mu, \mathrm{LTP} \text {,ins }}$ of the predicted $i n$ stantaneous proper motion for large $\Delta t$ is given by

$\varepsilon_{\mu, \mathrm{LTP}, \mathrm{ins}}^{2}=\varepsilon_{\mu, \mathrm{LTP}, \mathrm{m}}^{2}+c_{\mu}^{2}$.

The equations above describe what we have called the "analytic" approach in Paper I. For the "numerical" approach, we can take over for the LTP solution the formulae given in Paper I for the single-star mode (SI) with the following changes: (1) we should not redetermine the parallax $p$ of the star. The determination of $p$ by HIPPARCOS requires instantaneous values of $x$ and $\mu$, not the "mean" LTP values. Formally, we set all correlations between $p$ and the other quantities (position, proper motion) equal to zero. (2) In the variance-covariance matrix $\mathbf{D}$ we replace in the diagonal line the HIPPARCOS values of $D_{\mathrm{H}, 11}=\varepsilon_{\alpha *, \mathrm{H}}^{2}\left(T_{\mathrm{H}}\right)$ by

$D_{\mathrm{H}, 11, \mathrm{LTP}}=\varepsilon_{\alpha *, \mathrm{H}}^{2}\left(T_{\mathrm{H}}\right)+c_{x}^{2}$,

and $\varepsilon_{\delta, \mathrm{H}}^{2}$ by $\varepsilon_{\delta, \mathrm{H}}^{2}+c_{x}^{2}, \varepsilon_{\mu, \alpha *, \mathrm{H}}^{2}$ by $\varepsilon_{\mu, \alpha *}^{2}+c_{\mu}^{2}$, and $\varepsilon_{\mu, \delta, \mathrm{H}}^{2}$ by $\varepsilon_{\mu, \delta, \mathrm{H}}^{2}+c_{\mu}^{2}$, respectively. No changes are made in the non-diagonal elements (except for decoupling the parallax as described above), i.e. all the covariances remain in the LTP as they were in the SI mode, e.g.

$D_{\mathrm{H}, 12, \mathrm{LTP}}=D_{\mathrm{H}, 12}=\varepsilon_{\alpha *, \mathrm{H}}\left(T_{\mathrm{H}}\right) \varepsilon_{\delta, \mathrm{H}}\left(T_{\mathrm{H}}\right) \rho_{\alpha \delta, \mathrm{H}}\left(T_{\mathrm{H}}\right)$.

\section{Short-term prediction (STP)}

In this section, we consider the other limiting case of the general solution $x_{\mathrm{p}}(t)$, namely the limit for $\Delta t=$ $t-T_{2} \rightarrow 0$. This "short-term prediction" $x_{\mathrm{STP}}(t)$ is valid for epochs close to $T_{2}$ (in the case of using HIPPARCOS: $T_{2} \sim 1991.25$ ).

For the case $\Delta t \rightarrow 0$, we use for the correlation function $\xi(\Delta t)$ and $\zeta(\Delta t)$ Taylor series in $\Delta t$, and keep only terms which are linear in $\Delta t$. From the Eqs. (54) and (55) of WPSA, we obtain for small values of $\Delta t=t-T_{2}$

$$
\xi(\Delta t) \sim \xi(0)
$$

$\zeta(\Delta t) \sim \eta(0) \Delta t$

In Eq. (63), we have made use of the differential relation (28) of WPSA. Although HIPPARCOS values are already averaged over about 3 years of observation, the use of Eq. (63) is justified according to numerical investigations carried out by M. Biermann (1996, unpublished, see also WPSA, Sect. 3.4).

Inserting Eqs. (62) and (63) into the Eqs. (22) and (23), we find for the short-term prediction

$$
\begin{aligned}
\gamma_{\mathrm{STP}}(t)=( & \left(\xi(0)+\varepsilon_{x, 1}^{2}\right)\left(\eta(0)+\varepsilon_{\mu, 1}^{2}+\varepsilon_{\mu, 2}^{2}\right) \\
& +\varepsilon_{\mu, 1}^{2}\left(T_{2}-T_{1}\right)^{2}\left(\eta(0)+\varepsilon_{\mu, 2}^{2}\right) \\
& \left.+\varepsilon_{\mu, 1}^{2} \varepsilon_{\mu, 2}^{2}\left(T_{2}-T_{1}\right)\left(t-T_{2}\right)\right) / N \\
B_{\mathrm{STP}}(t)= & \beta_{\mathrm{STP}}(t)\left(t-T_{2}\right)=\left(\varepsilon_{\mu, 1}^{2} \varepsilon_{x, 2}^{2}\left(T_{2}-T_{1}\right)\right. \\
& +\left(\eta(0)\left(\xi(0)+\varepsilon_{x, 1}^{2}+\varepsilon_{x, 2}^{2}+\varepsilon_{\mu, 1}^{2}\left(T_{2}-T_{1}\right)^{2}\right)\right. \\
& \left.\left.+\varepsilon_{\mu, 1}^{2}\left(\xi(0)+\varepsilon_{x, 1}^{2}+\varepsilon_{x, 2}^{2}\right)\right)\left(t-T_{2}\right)\right) / N
\end{aligned}
$$

where $N$ is still given by Eq. (24). Inserting $\gamma_{\mathrm{STP}}(t)$ and $B_{\mathrm{STP}}(t)$ into Eq. (1), we obtain

$x_{\mathrm{STP}}(t)=x_{\mathrm{STP}}\left(T_{2}\right)+\mu_{\mathrm{STP}}\left(t-T_{2}\right)$,

which is a linear function of the epoch $t$. We do not use here a "central" epoch $T_{\mathrm{STP}}$, since our Taylor series for $x_{\mathrm{STP}}(t)$ is centered around $t=T_{2}$. The proper motion $\mu_{\text {STP }}$ can be derived as the sum of the coefficients in front of $t$ in the Eqs. (1), (63), and (64):

$$
\begin{aligned}
\mu_{\mathrm{STP}}= & \mu_{1} \\
& +\left(\left(x_{2}\left(T_{2}\right)-x_{1}\left(T_{1}\right)-\mu_{1}\left(T_{2}-T_{1}\right)\right) \varepsilon_{\mu, 1}^{2}\right. \\
& \left.\varepsilon_{\mu, 2}^{2}\left(T_{2}-T_{1}\right)\right) / N \\
& +\left(\mu_{2}-\mu_{1}\right)(\eta(0)(\xi(0) \\
& \left.+\varepsilon_{x, 1}^{2}+\varepsilon_{x, 2}^{2}+\varepsilon_{\mu, 1}^{2}\left(T_{2}-T_{1}\right)^{2}\right) \\
& \left.+\varepsilon_{\mu, 1}^{2}\left(\xi(0)+\varepsilon_{x, 1}^{2}+\varepsilon_{x, 2}^{2}\right)\right) / N
\end{aligned}
$$

After some algebra, $\mu_{\mathrm{STP}}$ can be rewritten as

$\mu_{\mathrm{STP}}=\frac{w_{\mu, 10, \mathrm{STP}} \mu_{10}+w_{\mu, 2} \mu_{2}}{w_{\mu, 10, \mathrm{STP}}+w_{\mu, 2}}$.

The "combined" mean proper motion $\mu_{10}$ is derived from $\mu_{1}$ and $\mu_{0}$ by

$\mu_{10}=\frac{w_{\mu, 1} \mu_{1}+w_{\mu, 0, \mathrm{LTP}} \mu_{0}}{w_{\mu, 1}+w_{\mu, 0, \mathrm{LTP}}}$.

$\mu_{0}, w_{\mu, 1}$, and $w_{\mu, 2}$ are given by the corresponding equations in Sect. 2.4, and $w_{\mu, 0, \mathrm{LTP}}$ by Eq. (53). The weight $w_{\mu, 10, \mathrm{STP}}$ of $\mu_{10}$ in the STP solution is given by

$w_{\mu, 10, \mathrm{STP}}=\frac{1}{\left(1 / w_{\mu, 10, \mathrm{LTP}}\right)+c_{\mu}^{2}}=\frac{1}{\varepsilon_{\mu, 10, \mathrm{LTP}}^{2}+c_{\mu}^{2}}$,

with

$w_{\mu, 10, \mathrm{LTP}}=w_{\mu, 1}+w_{\mu, 0, \mathrm{LTP}}=\frac{1}{\varepsilon_{\mu, 10, \mathrm{LTP}}^{2}}$ 
The meaning of Eqs. (68)-(71) is the following: $\mu_{\mathrm{STP}}$ is the weighted mean of $\mu_{2}$ and the combined mean proper motion $\mu_{10}$, where the cosmic error $c_{\mu}$ in $\mu_{10}$ has to be taken into account in $w_{\mu, 10, \mathrm{STP}}$. The combined mean proper motion $\mu_{10}$ is itself a weighted mean of $\mu_{1}$ and $\mu_{0}$, where for $\mu_{0}$ the cosmic error $c_{x}$ in $x_{2}\left(T_{2}\right)$ has to be included into $w_{\mu, 0, \mathrm{LTP}}$.

The exact expression for $x_{\mathrm{STP}}\left(T_{2}\right)$ is given by

$$
\begin{aligned}
x_{\mathrm{STP}}\left(T_{2}\right)= & x_{2}\left(T_{2}\right)-\varepsilon_{x, 2}^{2}\left(\left(\eta(0)+\varepsilon_{\mu, 1}^{2}+\varepsilon_{\mu, 2}^{2}\right)\right. \\
& \left(x_{2}\left(T_{2}\right)-x_{1}\left(T_{1}\right)-\mu_{1}\left(T_{2}-T_{1}\right)\right) \\
& \left.-\varepsilon_{\mu, 1}^{2}\left(T_{2}-T_{1}\right)\left(\mu_{2}\left(T_{2}\right)-\mu_{1}\right)\right) / N .
\end{aligned}
$$

For all practical purposes we can, however, neglect the last term in Eq. (72), which is proportional to $\varepsilon_{x, 2}^{2}$. The measuring accuracy $\varepsilon_{x, 2}$ for the HIPPARCOS positions $x_{2}\left(T_{2}\right)$ at $T_{2} \sim 1991.25$ is so high, relative to the other measuring errors and cosmic errors, that this term is usually of the order of 0.01 mas only. We use instead of Eq. (72) the very good approximation

$x_{\mathrm{STP}}\left(T_{2}\right)=x_{2}\left(T_{2}\right)$.

The mean errors of $x_{\mathrm{STP}}\left(T_{2}\right)$ and of $\mu_{\mathrm{STP}}$ are given by

$\varepsilon_{x, \operatorname{STP}}^{2}\left(T_{2}\right)=\varepsilon_{x, 2}^{2}\left(T_{2}\right)$,

$\varepsilon_{\mu, \mathrm{STP}}^{2}=\frac{1}{w_{\mu, 10, \mathrm{STP}}+w_{\mu, 2}}$.

The full uncertainty $\varepsilon_{x, \mathrm{STP}}(t)$ of $x_{\mathrm{STP}}(t)$ is given by

$$
\begin{aligned}
\varepsilon_{x, \mathrm{STP}}^{2}(t)= & \varepsilon_{x, \mathrm{STP}}^{2}\left(T_{2}\right)+\varepsilon_{\mu, \mathrm{STP}}^{2}\left(t-T_{2}\right)^{2} \\
& +\frac{1}{4} \xi_{0}^{(I V)}\left(t-T_{2}\right)^{4} .
\end{aligned}
$$

In Eq. (76), we have neglected the very small correlation between $x_{\mathrm{STP}}\left(T_{2}\right)=x_{2}\left(T_{2}\right)$ and $\mu_{\mathrm{STP}}$ which is caused by the use of $x_{2}\left(T_{2}\right)$ in deriving $\mu_{0}$ which in turn enters into $\mu_{\mathrm{STP}}$. The last term in Eq. (76) is the statistical uncertainty of a prediction based on instantaneous data at $T_{2}$ for small epoch differences $\Delta t$ (see Eq. (57) of WPSA). $\xi_{0}^{(\mathrm{IV})}$ is the fourth derivative of the correlation function $\xi(\Delta t)$ with respect to $\Delta t$ at $\Delta t=0$.

In the numerical approach for deriving the short-term prediction, we modify the procedure for the single-star mode presented in Paper I more strongly than for the LTP solution. As "observations" $\mathbf{b}$ we use first $\mathbf{b}_{\mathrm{H}}\left(T_{\mathrm{H}}\right)$, as in Paper I, the parallax $p_{\mathrm{H}}$ inclusive. The corresponding part $\mathbf{D}_{\mathrm{H}}$ of the variance-covariance matrix $\mathbf{D}$ remains also unchanged. The second part of $\mathbf{b}$, which we now call $\mathbf{b}_{\mathrm{m}}$ is given by the $\alpha_{*}$ and $\delta$ components of the combined mean proper motion $\mu_{10}$. The quantity $\mu_{10}$ is obtained in a preparatory step from Eq. (69), and its mean error from Eq. $(70)$ as $\left(w_{\mu, 10, \mathrm{STP}}\right)^{-1 / 2}$. Each component of $\mu_{10}$, i.e. of $\mathbf{b}_{\mathrm{m}}$, is considered not to be correlated with any other component of $\mathbf{b}$. The numerical approach for STP produces values for $x_{\mathrm{STP}}$ and $\mu_{\mathrm{STP}}$ (in $\alpha_{*}$ and $\delta$ ), a new parallax $p_{\mathrm{STP}}$, and the corresponding variance-covariance matrix.
In presenting the results in printed form, we use again central epochs $T_{\mathrm{STP}}$ (different for $\alpha_{*}$ and $\delta$ ), at which $x_{\mathrm{STP}}$ and $\mu_{\mathrm{STP}}$ are uncorrelated. However, $T_{\mathrm{STP}}$ differs usually only very slightly from the individual central epochs of the basic HIPPARCOS data.

\section{Transition from the short-term prediction to the long-term prediction}

\subsection{Transition in position}

As discussed in Sect. 2.4 and illustrated in Fig. 2, the general solution $x_{\mathrm{p}}(t)$ is a smooth transition from the shortterm prediction $x_{\mathrm{STP}}(t)$ for epochs $t$ close to $T_{2}$ to the long-term prediction $x_{\mathrm{LTP}}(t)$ for $t \rightarrow \pm \infty$. We are now asking for the "transition function" $\beta_{\text {trans }}(t)$ which describes this transition in $x$ :

$x_{\mathrm{p}}(t)=\left(1-\beta_{\text {trans }}(t)\right) x_{\mathrm{LTP}}(t)+\beta_{\text {trans }}(t) x_{\mathrm{STP}}(t)$.

Formally we can always solve Eq. (77) for $\beta_{\text {trans }}(t)$, using our former results for $x_{\mathrm{p}}(t), x_{\mathrm{LTP}}(t)$, and $x_{\mathrm{STP}}(t)$. However, the resulting transition function $\beta_{\text {trans }}(t)$ is then very complicated and depends unfortunately not only on the correlation functions, but also explicitely on the measured values of $x_{1}, x_{2}, \mu_{1}, \mu_{2}$, and on their measuring errors. Such a result is not very suitable for a practical application.

There does exist, however, an approximate treatment for the transition function $\beta_{\text {trans }}(t)$ which gives a very simple and easily applicable result, and which nevertheless describes the transition quantitatively rather accurately. The basic idea is the observation that in real applications the mean position $x_{1}\left(T_{1}\right)$ enters into the final result $x_{\mathrm{p}}(t)$ mainly through the proper motion $\mu_{0}$. This is caused by the small error of the HIPPARCOS position $x_{2}\left(T_{2}\right)$ with respect to the error of the mean (FK5 or GC) position $x_{1}\left(T_{1}\right)$. Only in cases of a large cosmic error $c_{x}(p)$ in $x_{2}\left(T_{2}\right)$, our approximation becomes less accurate. We therefore consider the transition function $\beta_{\text {trans }}(t)$ for the limiting case in which $\varepsilon_{x, 1}$ tends towards infinity while $\varepsilon_{\mu, 0}$ remains finite (equal to its actual value). The latter can be enforced by setting $T_{2}-T_{1}=\varepsilon_{x, 1} / \varepsilon_{\mu, 0}$. This means that we let go $T_{1} \rightarrow-\infty$ and $\varepsilon_{x, 1} \rightarrow+\infty$ in such a way that $\varepsilon_{\mu, 0}$ remains constant.

If we use this special case as an approximation, we derive after some lengthy algebra the following rather simple expression for the transition function:

$\beta_{\text {trans }}(t)=\frac{\zeta\left(t-T_{2}\right)}{\eta(0)\left(t-T_{2}\right)}$.

A similar function has already been derived as Eq. (70) in Sect. 4.2.4 of WPSA. The function $\beta_{\text {trans }}$ has the welcome property that it does neither depend on the measured values of $x$ and $\mu$ nor on the mean errors of a given star. These values are fully absorbed in the individual solutions $x_{\mathrm{STP}}(t)$ and $x_{\mathrm{LTP}}(t)$. Hence $\beta_{\text {trans }}(t)$ is the same function for all the objects. We should remark here that the apparently more complicated form of the transition function 
Table 1. Transition functions $\beta_{\text {trans }}(t)$ and $\nu_{\text {trans }}(t)$ for a simple example

\begin{tabular}{ccc}
\hline $\begin{array}{c}|\Delta t|=\left|t-T_{2}\right| \\
{[\text { years }]}\end{array}$ & $\beta_{\text {trans }}(\Delta t)$ & $\nu_{\text {trans }}(\Delta t)$ \\
\hline 0 & 1.000 & 1.000 \\
1 & 0.978 & 0.934 \\
2 & 0.915 & 0.752 \\
3 & 0.819 & 0.491 \\
4 & 0.700 & 0.202 \\
5 & 0.573 & -0.065 \\
6 & 0.449 & -0.270 \\
7 & 0.336 & -0.397 \\
8 & 0.241 & -0.445 \\
9 & 0.165 & -0.430 \\
10 & 0.108 & -0.373 \\
11 & 0.068 & -0.297 \\
12 & 0.041 & -0.219 \\
13 & 0.023 & -0.152 \\
14 & 0.013 & -0.099 \\
15 & 0.007 & -0.060 \\
20 & 0.0001 & -0.002 \\
\hline
\end{tabular}

according to Eq. (75) of WPSA is caused by the fact that we have used in WPSA a slightly different definition of the long and short-term prediction, namely $x_{2}(t)$ instead of $x_{\mathrm{STP}}(t)$ and $x_{1}(t)$ instead of $x_{\mathrm{LTP}}(t)$.

The function $\beta_{\text {trans }}(t)$ has the desired properties in the limits $\Delta t \rightarrow 0$ or $\infty$. For $t=T_{2}$, we have $\beta_{\text {trans }}\left(T_{2}\right)=1$, because of $\zeta(\Delta t) \sim \eta(0) \Delta t$ for small values of $\Delta t$. For $t \rightarrow \infty, \zeta\left(t-T_{2}\right)$ vanishes and hence $\beta_{\text {trans }}(\infty)=0$. An example for the full run of $\beta_{\text {trans }}\left(t-T_{2}\right)$, from 1 to 0 , is illustrated by the full curve shown in Fig. 8 of WPSA. Table 1 gives $\beta_{\text {trans }}\left(t-T_{2}\right)$ for a few values of $\left|t-T_{2}\right|$, using the example given in Sect. 3.6 of WPSA for the correlation function $\zeta(\Delta t)$. At $\left|t-T_{2}\right| \sim 5-6$ years, the general solution $x_{\mathrm{p}}(t)$ is about half-way between $x_{\mathrm{STP}}(t)$ and $x_{\mathrm{LTP}}(t)$. From both the Fig. 8 of WPSA and Table 1, we get an indication for the range of applicability of the short and long-term prediction. The short-term prediction $x_{\mathrm{STP}}(t)$ has a rather limited range of applicability, namely a few years around $T_{2}$ only. The long-term prediction $x_{\text {LTP }}(t)$ is a good approximation for the general solution $x_{\mathrm{p}}(t)$ for epoch differences $\left|t-T_{2}\right|$ which are larger than about 10 years. Hence the transition from STP to LTP is rather rapid, at least in our example.

We should remark here that (by chance) the transition function $\beta_{\text {trans }}(t)$ given by Eq. (78) is even strictly valid (exact) for the example used for the correlation functions in Sect. 3.6 of WPSA and adopted in Table 1. For more general runs of the correlation functions, $\beta_{\text {trans }}$ can become quite large at epochs around the "crossing time" at which $x_{\mathrm{LTP}}=x_{\mathrm{STP}}$, if $x_{\mathrm{LTP}}$ and $x_{\mathrm{STP}}$ are significantly different from $x_{\mathrm{p}}$ at that time.

\subsection{Transition in proper motion}

The most accurate prediction $\mu_{\mathrm{p}}(t)$ for the instantaneous proper motion $\mu(t)$ at an arbitrary epoch $t$ is formally given by

$$
\mu_{\mathrm{p}}(t)=\dot{x}_{\mathrm{p}}(t)=\left(1-\nu_{\text {trans }}(t)\right) \mu_{\mathrm{LTP}}+\nu_{\text {trans }}(t) \mu_{\mathrm{STP}} .
$$

The transition function $\nu_{\text {trans }}(t)$ for the proper motion $\mu$ is, similar to $\beta_{\text {trans }}$, in general a rather complicated function, which depends on the measured values of $\mu_{1}, \mu_{2}$, and $\mu_{0}$. If we adopt the same approximation as used in Sect. 5.1 for obtaining Eq. (78) for $\beta_{\text {trans }}$, namely $\varepsilon_{x, 1} \rightarrow$ $\infty$ while keeping $\varepsilon_{\mu, 0}$ constant, we derive for $\nu_{\text {trans }}(t)$ also a very simple expression:

$$
\begin{aligned}
\nu_{\text {trans }}(t) & =\beta_{\text {trans }}(t)+\dot{\beta}_{\text {trans }}(t)\left(t-T_{\mathrm{STP}}\right) \\
& =\frac{\eta\left(t-T_{\mathrm{STP}}\right)}{\eta(0)} .
\end{aligned}
$$

This transition function $\nu_{\text {trans }}(t)$ for $\mu(t)$ has the same proper limiting values as $\beta_{\text {trans }}(t)$ : for $t \rightarrow \infty$, we have $\nu_{\text {trans }}(\infty)=0$ and hence $\mu_{\mathrm{p}}(\infty)=\mu_{\mathrm{LTP}}$, and for $t=T_{\mathrm{STP}}$ we obtain $\nu_{\text {trans }}\left(T_{\mathrm{STP}}\right)=1$ and $\mu_{\mathrm{p}}\left(T_{\mathrm{STP}}\right)=\mu_{\mathrm{STP}}$. In Table 1 we list $\nu_{\text {trans }}(\Delta t)$ for a few values of $|\Delta t|=\mid t-$ $T_{\mathrm{STP}} \mid$, using Eq. (80) and the simple example for $\eta(\Delta t)$ given in Sect. 3.6 of WPSA.

The transition functions $\beta_{\text {trans }}(t)$ and $\nu_{\text {trans }}(t)$ according to Eqs. (78) and (80) have another nice property: if we use the example given in Sect. 3.6 of WPSA, then the function $\beta_{\text {trans }}$ depends only on $t-T_{2}$, but not on the individual cosmic error $c_{\mu}(p)=(\eta(0, p))^{1 / 2}$, since $\eta(0)$ occurs also as a factor in $\zeta\left(t-T_{2}\right)$ and cancels out in Eq. (78). Similarly, the transition function $\nu_{\text {trans }}(t)$ is a "scaled" function and depends on $t-T_{2}$ only, but not on $\eta(0, p)$.

\section{Cosmic errors}

For deriving the solutions in the LTP and STP mode, we need to know the cosmic errors $c_{\mu}=(\eta(0))^{1 / 2}$ and $c_{x}=(\xi(0))^{1 / 2}$. The cosmic errors depend strongly on the distance $r$ of the star from the Sun, or equivalently on the stellar parallax $p$. For 1202 "apparently single stars" from the basic FK5, we have obtained the functions $c_{\mu}(p)$ and $c_{x}(p)$ empirically by using groups of stars in various distance intervals. The data can be represented by the following fit functions:

$c_{\mu}(p)=(\eta(0, p))^{1 / 2}=\left(\frac{C_{1} p}{\left(C_{2}^{2}+p^{2}\right)^{1 / 2}}\right)^{1 / 2}$,
$c_{x}(p)=(\xi(0, p))^{1 / 2}=C_{3} c_{\mu}(p)$,

with

$C_{1}=9.30(\text { mas } / \text { year })^{2}$,

$C_{2}=22.14$ mas ,

$C_{3}=5.93$ years.

These versions of $c_{\mu}(p)$ and $c_{x}(p)$ have been used for the FK6 (Wielen et al. 1999d, 2000a). A table for $c_{\mu}$ and $c_{x}$ 
Table 2. Results of the combination method for the star HIP $9884=$ FK $74=$ GC $2538=\alpha$ Ari for the various modes

\begin{tabular}{|c|c|c|c|c|c|c|c|c|}
\hline \multirow[b]{2}{*}{ Quantity } & \multicolumn{4}{|c|}{ FK5 + HIPPARCOS } & \multicolumn{4}{|c|}{ GC + HIPPARCOS } \\
\hline & $\alpha_{*}$ & mean error & $\delta$ & mean error & $\alpha_{*}$ & mean error & $\delta$ & mean error \\
\hline \multicolumn{9}{|l|}{ Input data: } \\
\hline $\begin{array}{l}\Delta x_{\mathrm{F}}\left(T_{\mathrm{F}}\right) \text { or } \Delta x_{\mathrm{GC}}\left(T_{\mathrm{GC}}\right) \\
\Delta \mu_{\mathrm{F}} \text { or } \Delta \mu_{\mathrm{GC}} \\
T_{\mathrm{F}} \text { or } T_{\mathrm{GC}}\end{array}$ & $\begin{array}{r}-7.83 \\
+0.49 \\
1947.84\end{array}$ & $\begin{array}{l} \pm 12.52 \\
\pm \quad 0.40\end{array}$ & $\begin{array}{r}+96.95 \\
-1.20 \\
1929.73\end{array}$ & $\begin{array}{lr} \pm & 15.30 \\
\pm & 0.38\end{array}$ & $\begin{array}{r}-2.64 \\
+1.69 \\
1892.60\end{array}$ & $\begin{array}{l} \pm 49.92 \\
\pm \quad 1.24\end{array}$ & $\begin{array}{r}+236.68 \\
-3.98 \\
1890.30\end{array}$ & $\begin{array}{l} \pm 32.88 \\
\pm \quad 1.86\end{array}$ \\
\hline $\begin{array}{l}\Delta x_{\mathrm{H}}\left(T_{\mathrm{H}, \text { ind }}\right) \\
\Delta \mu_{\mathrm{H}} \\
T_{\mathrm{H}, \text { ind }}\end{array}$ & $\begin{array}{r}0.00 \\
0.00 \\
1991.26\end{array}$ & $\begin{array}{l} \pm 0.77 \\
\pm 1.01\end{array}$ & $\begin{array}{r}0.00 \\
0.00 \\
1991.51\end{array}$ & $\begin{array}{l} \pm 0.54 \\
\pm 0.77\end{array}$ & $\begin{array}{r}0.00 \\
0.00 \\
1991.26\end{array}$ & $\begin{array}{l} \pm 0.77 \\
\pm 1.01\end{array}$ & $\begin{array}{r}0.00 \\
0.00 \\
1991.51\end{array}$ & $\begin{array}{l} \pm 0.54 \\
\pm 0.77\end{array}$ \\
\hline $\begin{array}{l}p_{\mathrm{H}} \\
c_{x} \\
c_{\mu}\end{array}$ & $\begin{array}{r}49.48 \\
17.28 \\
2.91\end{array}$ & \pm 0.99 & $\begin{array}{r}17.28 \\
2.91\end{array}$ & & $\begin{array}{r}49.48 \\
17.28 \\
2.91\end{array}$ & \pm 0.99 & $\begin{array}{r}17.28 \\
2.91\end{array}$ & \\
\hline$\Delta \mu_{0}$ & +0.18 & \pm 0.29 & -1.57 & \pm 0.25 & +0.03 & \pm 0.51 & -2.34 & \pm 0.32 \\
\hline \multicolumn{9}{|c|}{ Results for the single-star mode SI: } \\
\hline $\begin{array}{l}\Delta x_{\mathrm{SI}}\left(T_{\mathrm{SI}}\right) \\
\Delta \mu_{\mathrm{SI}} \\
T_{\mathrm{SI}}\end{array}$ & $\begin{array}{r}-0.18 \\
+0.23 \\
1991.12\end{array}$ & $\begin{array}{l} \pm 0.76 \\
\pm 0.23\end{array}$ & $\begin{array}{r}+0.14 \\
-1.34 \\
1991.47\end{array}$ & $\begin{array}{l} \pm 0.54 \\
\pm 0.20\end{array}$ & $\begin{array}{r}-0.23 \\
+0.04 \\
1991.26\end{array}$ & $\begin{array}{l} \pm 0.77 \\
\pm 0.42\end{array}$ & $\begin{array}{r}+0.08 \\
-2.00 \\
1991.51\end{array}$ & $\begin{array}{l} \pm 0.54 \\
\pm 0.29\end{array}$ \\
\hline$\Delta p_{\mathrm{SI}}$ & -0.57 & \pm 0.95 & & & -0.98 & \pm 0.95 & & \\
\hline $\begin{array}{l}\text { Results for the long-term } \\
\Delta x_{\mathrm{LTP}}\left(T_{\mathrm{LTP}}\right) \\
\Delta \mu_{\mathrm{LTP}} \\
T_{\mathrm{LTP}}\end{array}$ & $\begin{array}{r}\text { mode I } \\
-5.14 \\
+0.36 \\
1962.77\end{array}$ & $\begin{array}{l}\text { LTP: } \\
\quad \pm 10.14 \\
\quad \pm 0.31\end{array}$ & $\begin{array}{r}+54.37 \\
-1.38 \\
1956.76\end{array}$ & $\begin{array}{r} \pm 11.46 \\
\pm 0.27\end{array}$ & $\begin{array}{r}-0.30 \\
+0.28 \\
1980.68\end{array}$ & $\begin{array}{r} \pm 16.34 \\
\pm 0.49\end{array}$ & $\begin{array}{r}+51.26 \\
-2.37 \\
1969.40\end{array}$ & $\begin{array}{r} \pm 15.30 \\
\pm 0.36\end{array}$ \\
\hline \multicolumn{9}{|c|}{ Results for the short-term prediction mode $\mathbf{S T P}$ : } \\
\hline $\begin{array}{l}\Delta x_{\mathrm{STP}}\left(T_{\mathrm{STP}}\right) \\
\Delta \mu_{\mathrm{STP}} \\
T_{\mathrm{STP}}\end{array}$ & $\begin{array}{r}-0.01 \\
0.00 \\
1991.26\end{array}$ & $\begin{array}{l} \pm 0.77 \\
\pm 0.95\end{array}$ & $\begin{array}{r}0.00 \\
-0.08 \\
1991.52\end{array}$ & $\begin{array}{l} \pm 0.54 \\
\pm 0.74\end{array}$ & $\begin{array}{r}-0.02 \\
-0.03 \\
1991.26\end{array}$ & $\begin{array}{l} \pm 0.77 \\
\pm 0.95\end{array}$ & $\begin{array}{r}0.00 \\
-0.15 \\
1991.52\end{array}$ & $\begin{array}{l} \pm 0.54 \\
\pm 0.74\end{array}$ \\
\hline$\Delta p_{\mathrm{STP}}$ & -0.03 & \pm 0.99 & & & -0.06 & \pm 0.99 & & \\
\hline
\end{tabular}

Units: mas, mas/year, or years.

as a function of $p$ or $r$, based in Eqs. (81)-(85), is given on page 12 of Wielen et al. (1999d).

In the future we hope to determine also the dependence of the cosmic errors on the absolute magnitude (or mass) of the stars for a given parallax. A comparison of the results for the cosmic errors based on the FK5 stars with those derived from the (on average fainter) GC stars seems to indicate a rather weak dependence on the brightness of the stars (Wielen et al. 1998).

\section{An example: $\alpha$ Ari}

In order to illustrate our combination method for all the three modes (SI, LTP, STP), we give in Table 2 the results for one individual star. As in Paper I (Sect. 5), we give the positions $x(t)$ and the proper motions $\mu(t)$ always relative to the HIPPARCOS solution as $\Delta x(t)=x(t)-x_{\mathrm{H}}(t)$ and $\Delta \mu(t)=\mu(t)-\mu_{\mathrm{H}}(t)$, in order to save printing space and to make the comparison of the results easier. The groundbased data are always reduced to the HIPPARCOS system. The results of the single-star mode presented here in Paper II differ somewhat from those of Paper I, because we now adopt slightly improved systematic differences FK5HIP and GC-HIP. For a valid comparison of the results of the three different modes it is necessary to use exactly the same basic input data.

Table 2 shows that the short-term prediction is usually quite close to the HIPPARCOS solution. On the other hand, the long-term prediction differs most strongly from the HIPPARCOS solution, since the HIPPARCOS values are entering with a lower weight into the LTP mode than in the SI mode, because of the cosmic errors in the HIPPARCOS data. The central epoch $T_{\text {LTP }}$ is the only one which is usually significantly earlier than $T_{\mathrm{H}} \sim 1991.25$, and the mean error of the central position $x_{\mathrm{LTP}}\left(T_{\mathrm{LTP}}\right)$ is typically only slightly smaller than the $\operatorname{cosmic}$ error $c_{x}(p)$. 


\section{Error budget}

In Table 3 we present the error budget of proper motions in the three different modes (SI, LTP, STP) for two samples of basic FK5 stars.

The mean errors $\varepsilon_{\mu}$ given in Table 3 refer to one "mean" coordinate component. It is obtained as an rms average over $\varepsilon_{\mu, \alpha *}$ and $\varepsilon_{\mu, \delta}$, and over all the stars in the corresponding sample. The error budget for the 1535 basic FK5 stars is slightly fictious, since this sample contains double stars for which the FK6 provides in reality "special" solutions instead of the "direct" combination solutions discussed in this paper. Nevertheless, the results for this sample provide a valid indication for the overall accuracy of our combination method in the direct modes SI, LTP, and STP. The sample of 1202 basic FK5 stars contains "apparently single objects" only. Most of these stars (878 objects) have direct solutions in the FK6. The error budget for these 878 basic FK5 stars in Part I of the FK6 is given in Wielen et al. (1999d). The error budgets for 3272 additional fundamental stars with direct solutions in the three modes are presented in Part III of the FK6 (Wielen et al. 2000a).

Table 4 gives the error budget for the combination of the GC (Boss et al. 1937) with HIPPARCOS. The sample of GC stars are the "full sample" of 29717 GC stars observed by HIPPARCOS, and the "subsample" of 11737 GC stars with linear HIPPARCOS standard solutions. From Table 4 it is obvious that the original proper motions $\mu_{\mathrm{GC}}$ of the $\mathrm{GC}$ do not contribute significantly to the GC+HIP on average. However, for some brighter and well-observed stars, the accuracy of $\mu_{\mathrm{GC}}$ is much better than the rms value of $\varepsilon_{\mu, \mathrm{GC}}$ seen in Table 4 . Furthermore, the proper motion $\mu_{0(\mathrm{GC}) \mathrm{H}}$, derived from the central positions of the GC and the HIPPARCOS Catalogue, has usually a quite reasonable accuracy for most of the GC stars.

The typical gain in accuracy in the proper motions derived in the long-term prediction mode, relative to the original HIPPARCOS proper motions, is a factor of 4.6 for the basic fundamental stars in the FK $6=\mathrm{FK} 5+\mathrm{HIP}$, and a factor of 1.8 for the $11773 \mathrm{GC}$ stars in the GC+HIP. This improvement in the LTP mode with respect to HIPPARCOS is a consequence of the cosmic errors in the instantaneously measured HIPPARCOS proper motions. In contrast to the LTP mode, the short-term predictions (STP mode) do not differ so much from the HIPPARCOS solutions. However, in most cases we are more interested in the long-term averaged proper motion (LTP) or in the single-mode result (SI), where the gain in accuracy is quite significant.

\section{Problems and applications}

The long-term predictions and the short-term predictions derived in the former sections are statistically the best astrometric solution for a sample of "apparently
Table 3. Error budget for FK6 proper motions

Typical mean errors of proper motions

(in one component, averaged over $\mu_{\alpha *}$ and $\mu_{\delta}$; units: mas/year)

\begin{tabular}{|c|c|c|c|c|}
\hline \multirow[t]{2}{*}{ Sample of stars: } & \multicolumn{2}{|c|}{$1535 \mathrm{FK}$} & \multicolumn{2}{|c|}{$1202 \mathrm{FK}$} \\
\hline & \multicolumn{3}{|c|}{ ms aver. median rms aver. } & median \\
\hline SI: & \multicolumn{3}{|c|}{ single-star mode } & \\
\hline $\begin{array}{l}\text { HIPPARCUS } \\
\text { random }\end{array}$ & 0.82 & 0.63 & 0.68 & 0.61 \\
\hline FK5 & & & & \\
\hline random & 0.76 & 0.64 & 0.77 & 0.67 \\
\hline system & 0.28 & 0.25 & 0.28 & 0.25 \\
\hline total & 0.81 & 0.70 & 0.83 & 0.72 \\
\hline$\mu_{0}$ & & & & \\
\hline random & 0.53 & 0.43 & 0.54 & 0.45 \\
\hline system & 0.24 & 0.23 & 0.25 & 0.23 \\
\hline total & 0.58 & 0.49 & 0.59 & 0.51 \\
\hline $\mathrm{FK} 6=\mathrm{FK} 5+\mathrm{HIP}$ & 0.35 & 0.33 & 0.35 & 0.34 \\
\hline $\begin{array}{c}\text { ratio of HIPPARCOS } \\
\text { to FK6 errors }\end{array}$ & 2.3 & 1.9 & 1.9 & 1.8 \\
\hline
\end{tabular}

\begin{tabular}{lllll}
\multicolumn{1}{c}{ LTP: } & \multicolumn{4}{l}{ long-term prediction } \\
$\begin{array}{l}\text { HIPPARCOS } \\
\quad \text { random }\end{array}$ & 0.82 & 0.63 & 0.68 & 0.61 \\
$\quad$ cosmic (in $\mu$ ) & 2.15 & 2.05 & 2.13 & 2.04 \\
$\quad$ total & 2.30 & 2.14 & 2.24 & 2.13 \\
FK5 & & & & \\
$\quad$ random & 0.76 & 0.64 & 0.77 & 0.67 \\
$\quad$ system & 0.28 & 0.25 & 0.28 & 0.25 \\
$\quad$ total & 0.81 & 0.70 & 0.83 & 0.72 \\
$\mu_{0} \quad$ & & & \\
$\quad$ random & 0.53 & 0.43 & 0.54 & 0.45 \\
$\quad$ system & 0.24 & 0.23 & 0.25 & 0.23 \\
$\quad$ cosmic (due to $x)$ & 0.32 & 0.30 & 0.32 & 0.30 \\
$\quad$ total & 0.66 & 0.57 & 0.68 & 0.59 \\
FK6 = FK5+HIP & 0.49 & 0.44 & 0.49 & 0.45 \\
ratio of HIPPARCOS & 4.7 & 4.9 & 4.6 & 4.7 \\
$\quad$ to FK6 errors & & & & \\
\hline$\quad$ & & &
\end{tabular}

\begin{tabular}{lllll}
\hline \multicolumn{1}{c}{ STP: } & \multicolumn{3}{l}{ short-term prediction } \\
$\begin{array}{l}\text { HIPPARCOS } \\
\quad \text { random }\end{array}$ & 0.82 & 0.63 & 0.68 & 0.61 \\
$\begin{array}{l}\text { FK5 } \\
\quad \text { random }\end{array}$ & 0.76 & 0.64 & 0.77 & 0.67 \\
$\quad$ system & 0.28 & 0.25 & 0.28 & 0.25 \\
$\quad$ cosmic (in $\mu$ ) & 2.15 & 2.05 & 2.13 & 2.04 \\
$\quad$ total & 2.30 & 2.16 & 2.28 & 2.16 \\
$\mu_{0}$ & & & & \\
$\quad$ random & 0.53 & 0.43 & 0.54 & 0.45 \\
$\quad$ system & 0.24 & 0.23 & 0.25 & 0.23 \\
$\quad$ cosmic (due to $x)$ & 0.32 & 0.30 & 0.32 & 0.30 \\
$\quad$ cosmic (in $\mu$ ) & 2.15 & 2.05 & 2.13 & 2.04 \\
$\quad$ total & 2.25 & 2.13 & 2.23 & 2.12 \\
FK6 = FK5+HIP & 0.68 & 0.59 & 0.62 & 0.57 \\
$\quad$ ratio of HIPPARCOS & 1.2 & 1.1 & 1.1 & 1.1 \\
$\quad$ to FK6 errors & & & & \\
& & & & \\
\hline
\end{tabular}


Table 4. Error budget for GC+HIP proper motions

Typical mean errors of proper motions (in one component, averaged over $\mu_{\alpha *}$ and $\mu_{\delta} ;$ units: mas/year)

\begin{tabular}{|c|c|c|c|c|}
\hline \multirow[t]{2}{*}{ Sample of stars: } & \multicolumn{2}{|c|}{$29717 \mathrm{GC}$} & \multicolumn{2}{|c|}{$11773 \mathrm{GC}$} \\
\hline & \multicolumn{2}{|c|}{ rms aver. median } & ns aver & median \\
\hline \multicolumn{5}{|c|}{ SI: $\quad$ single-star mode } \\
\hline $\begin{array}{l}\text { HIPPARCOS } \\
\text { random }\end{array}$ & 1.47 & 0.73 & 0.75 & 0.69 \\
\hline \multicolumn{5}{|l|}{ GC } \\
\hline random & 10.57 & 9.38 & 8.59 & 7.55 \\
\hline system & 0.57 & 0.49 & 0.56 & 0.48 \\
\hline total & 10.59 & 9.39 & 8.61 & 7.57 \\
\hline \multicolumn{5}{|l|}{$\mu_{0}$} \\
\hline random & 1.78 & 1.74 & 1.42 & 1.44 \\
\hline system & 0.18 & 0.12 & 0.13 & 0.12 \\
\hline total & 1.79 & 1.75 & 1.43 & 1.45 \\
\hline $\mathrm{GC}+\mathrm{HIP}$ & 0.72 & 0.63 & 0.62 & 0.58 \\
\hline $\begin{array}{l}\text { ratio of HIPPARCOS } \\
\text { to GC+HIP errors }\end{array}$ & 2.0 & 1.2 & 1.2 & 1.2 \\
\hline
\end{tabular}

\begin{tabular}{lrlll}
\multicolumn{1}{c}{ LTP: } & long-term prediction & \\
HIPPARCOS & & & & \\
$\quad$ random & 1.47 & 0.73 & 0.75 & 0.69 \\
$\quad$ cosmic (in $\mu$ ) & 1.75 & 1.60 & 1.80 & 1.66 \\
$\quad$ total & 2.29 & 1.76 & 1.95 & 1.80 \\
GC & & & & \\
$\quad$ random & 10.57 & 9.38 & 8.59 & 7.55 \\
$\quad$ system & 0.57 & 0.49 & 0.56 & 0.48 \\
$\quad$ total & 10.59 & 9.39 & 8.61 & 7.57 \\
$\mu_{0}$ & & & & \\
$\quad$ random & 1.78 & 1.74 & 1.42 & 1.44 \\
$\quad$ system & 0.18 & 0.12 & 0.13 & 0.12 \\
$\quad$ cosmic (due to $x$ ) & 0.12 & 0.10 & 0.12 & 0.11 \\
$\quad$ total & 1.79 & 1.75 & 1.43 & 1.45 \\
GC+HIP & 1.17 & 1.15 & 1.06 & 1.05 \\
ratio of HIPPARCOS & 2.0 & 1.5 & 1.8 & 1.7 \\
$\quad$ to GC+HIP errors & & & & \\
\hline
\end{tabular}

\section{STP: $\quad$ short-term prediction}

\section{HIPPARCOS}

$\begin{array}{lrrrl}\quad \text { random } & 1.47 & 0.73 & 0.75 & 0.69 \\ \text { GC } & & & & \\ \quad \text { random } & 10.57 & 9.38 & 8.59 & 7.55 \\ \quad \text { system } & 0.57 & 0.49 & 0.56 & 0.48 \\ \quad \text { cosmic (in } \mu \text { ) } & 1.75 & 1.60 & 1.80 & 1.66 \\ \quad \text { total } & 10.73 & 9.53 & 8.79 & 7.75 \\ \mu_{0} & & & & \\ \quad \text { random } & 1.78 & 1.74 & 1.42 & 1.44 \\ \text { system } & 0.18 & 0.12 & 0.13 & 0.12 \\ \text { cosmic (due to } x) & 0.12 & 0.10 & 0.12 & 0.11 \\ \quad \text { cosmic (in } \mu \text { ) } & 1.75 & 1.60 & 1.80 & 1.66 \\ \text { total } & 2.51 & 2.37 & 2.30 & 2.20 \\ \text { GC+HIP } & 0.82 & 0.69 & 0.69 & 0.65 \\ \text { ratio of HIPPARCOS } & 1.8 & 1.1 & 1.1 & 1.1\end{array}$

single-stars". However, an unsatisfactory property of these statistically valid solutions is the fact that we are not able to make proper use of the available information on the individual behaviour of the stars. For example, we use the overall cosmic errors $c_{\mu}(p)$ and $c_{x}(p)$, no matter whether the object is a $\Delta \mu$ binary or a single-star candidate (Wielen et al. 1999a). In principle, for each individual star, one would have to use "conditioned correlation functions" which are based on the individually observed differences between the instantaneous measurements and the mean data (e.g. on $\left.\mu_{\mathrm{FK} 5}-\mu_{\mathrm{HIP}}\right)$. Unfortunately, the conditioned correlation functions, i.e. "conditioned cosmic errors" in particular, are not available at present.

We should also point out that presently the cosmic errors $c_{x}$ in position are much more uncertain than the cosmic errors $c_{\mu}$ in proper motion. While the typical values of $c_{\mu}$ are larger than the measuring errors $\varepsilon_{\mu, \mathrm{FK} 5}, \varepsilon_{\mu, 0}$, and $\varepsilon_{\mu, \mathrm{HIP}}$, the typical values of $c_{x}$ are nearly lost in the measuring errors of the ground-based data.

Furthermore, our knowledge about the actual form of the correlation functions $\xi(\Delta t), \eta(\Delta t), \zeta(\Delta t)$ for $\Delta t \neq 0$ is presently still quite rudimentary. The simple example for the correlation functions presented in Sect. 3.6 of WPSA is mainly given for illustrating the general behaviour of these functions. The application of this example to real data should be done very cautiously. Hence the real transition from the STP solution to the LTP solution (discussed in Sect. 5) is quantitatively not well-determined.

Which of the three solutions offered (SI, LTP, STP) should be used in real applications? For single-star candidates, the single-star mode should be adopted, although some of the single-star candidates may nevertheless be binaries. If the user is handling a sample of "apparently single stars" (which usually contains single-star candidates, $\Delta \mu$ binaries, and intermediate cases), then the LTP or STP solutions are recommended, depending on the corresponding epoch difference $\Delta t=t-T_{\mathrm{H}}\left(\right.$ with $\left.T_{\mathrm{H}} \sim 1991\right)$.

The problems discussed above are especially severe for objects detected as $\Delta \mu$ binaries (Wielen et al. 1999a). For $\Delta \mu$ binaries the difference between the instantaneous proper motion $\left(\mu_{\mathrm{HIP}}\right)$ and the mean one (e.g., $\mu_{\mathrm{FK} 5}$ or $\left.\mu_{0}\right)$ is sometimes much larger than the cosmic error $c_{\mu}$ expected on average. In such a case the weight $w_{\mu, 2, \mathrm{LTP}}$ (Eq. $(53))$ of the HIPPARCOS proper motion $\left(\mu_{\mathrm{HIP}}=\mu_{2}\right)$ is higher than appropriate for this individual object. The derived mean proper motion $\mu_{\mathrm{LTP}}$ is then biased towards the HIPPARCOS proper motion, and the derived mean error $\varepsilon_{\mu, \mathrm{LTP}}$ is too small. In the case of extreme $\Delta \mu$ binaries, it is better to adopt a properly weighted mean of $\mu_{1}$ (e.g. $\left.\mu_{\mathrm{FK} 5}\right)$ and $\mu_{0}$ as a prediction for the mean proper motion $\mu_{\mathrm{m}}$. The main problem in such a procedure is the unknown individual value of the cosmic error in the HIPPARCOS position, which is higher than the value of $c_{x}$ expected on average. This value enters into the weight of $\mu_{0}$, and hence into the predicted value of $\mu_{\mathrm{m}}$. We shall discuss this problem in more detail in a subsequent paper. In any case, the LTP solutions (and, of course, the SI solutions) for $\Delta \mu$ binaries are inherently the least accurate 
ones among the class of direct solutions, because of the disturbing double-star nature of these objects.

\section{Summary and outlook}

In this Paper II, we have derived and discussed an appropriate method to combine a ground-based astrometric catalogue (such as the FK5 or GC) with the HIPPARCOS Catalogue, taking cosmic errors (due to undetected binaries) in the quasi-instantaneously measured data into account. The method leads to long-term predictions (LTP mode) and to short-term predictions (STP mode), which are the limiting cases of the general solution. The general solution is a smooth transition from the STP to the LTP mode. The case of single stars with no cosmic errors was already treated in Paper I (SI mode). In a subsequent paper, we shall present "special solutions" for known double stars.

\section{References}

Bien, R., Fricke, W., Lederle, T., \& Schwan, H. 1978, Veröff. Astron. Rechen-Inst. Heidelberg, No. 29

Boss, B., Albrecht, S., Jenkins, H., et al. 1937, General Catalogue of 33342 Stars for the Epoch 1950, Carnegie Institution of Washington, Publ., No. 486 [GC]

ESA 1997, The Hipparcos Catalogue, ESA SP-1200

Fricke, W., Schwan, H., Lederle, T., et al. 1988, Veröff. Astron. Rechen-Inst. Heidelberg, No. 32 [FK5, Part I]

Fricke, W., Schwan, H., Corbin, T., et al. 1991, Veröff. Astron. Rechen-Inst. Heidelberg, No. 33 [FK5, Part II]

Wielen, R. 1995a, A\&A, 302, 613
Wielen, R. 1995b, in Future Possibilities for Astrometry in Space, ed. M. A. C. Perryman, \& F. van Leeuwen, ESA SP-379, 65

Wielen, R. 1997, A\&A, 325, 367 [WPSA]

Wielen, R., Schwan, H., Dettbarn, C., Jahreiß, H., \& Lenhardt, H. 1997, in Hipparcos Venice '97, Presentation of the Hipparcos and Tycho Catalogues and first astrophysical results of the Hipparcos space astrometry mission, ed. B. Battrick, M. A. C. Perryman, \& P. L. Bernacca, ESA SP402, 727

Wielen, R., Schwan, H., Dettbarn, C., Jahreiß, H., \& Lenhardt, H. 1998, in The Message of the Angles - Astrometry from 1798 to 1998, Proceedings of the International Spring Meeting of the Astronomische Gesellschaft, held in Gotha, 11-15 May 1998, ed. P. Brosche, W. R. Dick, O. Schwarz, \& R. Wielen, Acta Historica Astronomiae, vol. 3, Verlag Harri Deutsch, Thun and Frankfurt am Main, 123

Wielen, R., Dettbarn, C., Jahreiß H., Lenhardt, H., \& Schwan, H. 1999a, A\&A, 346, 675

Wielen, R., Lenhardt, H., Schwan, H., \& Dettbarn, C. 1999b, A\&A, 347, 1046, Paper I

Wielen, R., Schwan, H., Dettbarn, C., Jahreiß, H., \& Lenhardt, H. 1999c, in Modern Astrometry and Astrodynamics, Proceedings of the International Conference honouring Heinrich Eichhorn, held at Vienna Observatory, Austria, 25-26 May 1998, ed. R. Dvorak, H. F. Haupt, \& K. Wodnar, Verlag der Österreichischen Akademie der Wissenschaften, Wien, 161

Wielen, R., Schwan, H., Dettbarn, C., et al. 1999d, Veröff. Astron. Rechen-Inst. Heidelberg, No. 35 [FK6, Part I]

Wielen, R., Schwan, H., Dettbarn, C., et al. 2000a, Veröff. Astron. Rechen-Inst. Heidelberg, No. 37 [FK6, Part III]

Wielen, R., Jahreiß, H., Dettbarn, C., Lenhardt, H., \& Schwan, H. 2000b, A\&A, 360, 399 